\title{
Role of Eye, Head, and Shoulder Geometry in the Planning of Accurate Arm Movements
}

\author{
D.Y.P. HENRIQUES ${ }^{1-3}$ AND J. D. CRAWFORD ${ }^{1-5}$ \\ ${ }^{1}$ Centre for Vision Research, ${ }^{2}$ Canadian Institutes of Health Research Group for Action and Perception, ${ }^{3}$ Department of \\ Psychology, ${ }^{4}$ Department of Biology, and ${ }^{5}$ Department of Kinesiology and Health Sciences, York University, Toronto, \\ Ontario M3J 1P3, Canada
}

Received 19 June 2001; accepted in final form 27 November 2001

Henriques, D.Y.P. and J. D. Crawford. Role of eye, head and shoulder geometry in the planning of accurate arm movements. J Neurophysiol 87: 1677-1685, 2002; 10.1152/jn.00509.2001. Eyehand coordination requires the brain to integrate visual information with the continuous changes in eye, head, and arm positions. This is a geometrically complex process because the eyes, head, and shoulder have different centers of rotation. As a result, head rotation causes the eye to translate with respect to the shoulder. The present study examines the consequences of this geometry for planning accurate arm movements in a pointing task with the head at different orientations. When asked to point at an object, subjects oriented their arm to position the fingertip on the line running from the target to the viewing eye. But this eye-target line shifts when the eyes translate with each new head orientation, thereby requiring a new arm pointing direction. We confirmed that subjects do realign their fingertip with the eyetarget line during closed-loop pointing across various horizontal head orientations when gaze is on target. More importantly, subjects also showed this head-position-dependent pattern of pointing responses for the same paradigm performed in complete darkness. However, when gaze was not on target, compensation for these translations in the rotational centers partially broke down. As a result, subjects tended to overshoot the target direction relative to current gaze; perhaps explaining previously reported errors in aiming the arm to retinally peripheral targets. These results suggest that knowledge of head position signals and the resulting relative displacements in the centers of rotation of the eye and shoulder are incorporated using open-loop mechanisms for eye-hand coordination, but these translations are best calibrated for foveated, gaze-on-target movements.

\section{IN T R O D U C T I O N}

The brain uses information about body geometry to guide movements. When you reach for an object, vision may reveal that your hand is $20^{\circ}$ right of its target, but this information alone cannot tell you how you should move your joints, or whether you should activate or relax various muscles. For that, you also need proprioceptive or motor information about the current angles of your eyeball, your head, and your arm joints, as well as stored data about the geometry of the bones and muscles in the linkage from eyeball to fingers. The brain must contain a representation, or model, of body geometry. How sophisticated is this body model?

Theoretically, the brain could get by with less accurate

Address for reprint requests: J. D. Crawford, Dept. of Psychology, York University, 4700 Keele St., Toronto, Ontario M3J 1P3, Canada (E-mail: jdc@yorku.ca). information about body geometry if it relied on visual feedback. It is a property of feedback systems that they can function adequately with suboptimal processors inside the feedback loop. This is in accordance with studies showing that many motor tasks are performed better with visual feedback (Helms Tillery et al. 1991; Prablanc et al. 1986; Rossetti et al. 1994; Van Donkelaar and Straub 2000). So the body model might be inexact; its deficiencies counteracted by visual correction of the hand's path. Alternatively, the brain might develop a highly sophisticated body model, refining it over many years of motor learning.

Here we study this question by having people perform a geometrically exacting motor task with and without visual feedback. The task we chose was straight-arm pointing, maybe the simplest example of hand-eye coordination, but geometrically complex all the same because it involves several movable parts (eye, head, clavicle, and arm) rotating about different centers. These noncoincident rotations have been largely ignored in studies of eye-hand coordination, but they are crucial for the underlying visuomotor transformations, not just for pointing but for most motor tasks.

Asked to point at an object, people do not orient the arm directly toward the target, but instead place the fingertip close to the line running from the target to the dominant eye, the eye-target line (Fig. 1A). Most people can verify this for themselves by pointing to something and then looking at it through each eye in turn. How does linkage geometry affect this task? Because the rotary centers of eye, head, and shoulder are spatially separated, any motion within the linkage alters the required relation between visual input and arm control. Any head turn transports the eyes, moving the eye-target line and therefore the desired location of the fingertip, as you can confirm by pointing at a distant target and then turning your head. Figure 1, $B$ and $C$, shows that quite different arm positions are needed when the head is turned $40^{\circ}$ left and $40^{\circ}$ right. Here we examine whether people adjust their pointing in this way to keep the finger on the eye-target line when the head turns, and whether they still do so in the absence of vision. If they can, the neural body model must be sophisticated enough to represent the complex eye-arm linkage with its multiple, mobile centers of rotation.

\footnotetext{
The costs of publication of this article were defrayed in part by the payment of page charges. The article must therefore be hereby marked "advertisement" in accordance with 18 U.S.C. Section 1734 solely to indicate this fact.
} 


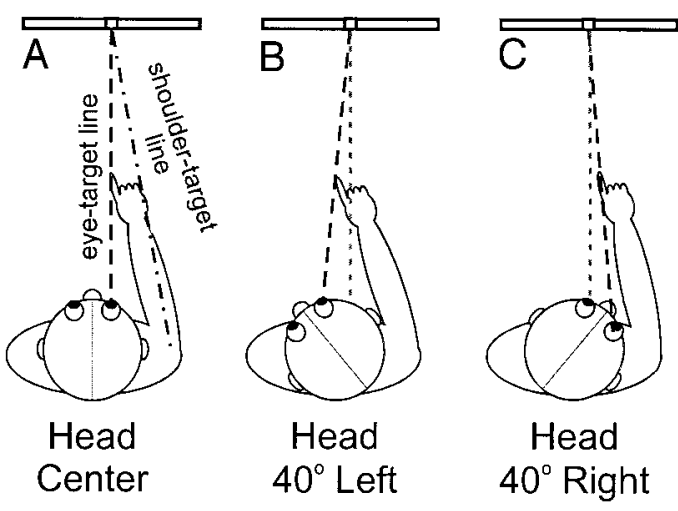

FIG. 1. Schematic illustration of how arm pointing direction toward a target varies with head position seen from an above view. $A-C$ : drawings show how subjects might point toward the same central target $(\square)$ at the 3 head orientations: straight ahead $(A), 40^{\circ}$ left $(B)$, and $40^{\circ}$ right $(C)$ of center. The central target is located directly in front of the right eye when the head is at center (not in front of the center of rotation of the head). The subject could point toward the target by orienting their fully extended arm and fingertip along a line projecting from the rotational center to the target (shoulder-target pointing) as shown by the dotted-dashed line in $A$. In this case, pointing direction would not depend on head position. Alternatively the arm may be oriented to place the fingertip on the line joining the eye and the target (eye-target line). The eye-target line (black dashed line) shifts when the eye translates with each new head position, so in this case horizontal arm position would differ, as shown in $A-C$ (compare the eye-targets line for the head positions in $B$ and $C$ with the light dashed lines representing the eye-target line for the central head position in $A$ ).

We also study whether subjects do the same when the eye is not aimed at the pointing target. People are less accurate in locating a remembered target when gaze is shifted away from it (Bock 1986, 1993; Enright 1995; Henriques et al. 1998). One of the goals of the present study is to quantify pointing errors produced when the subject deviates their head along with gaze, and to investigate how such errors might arise.

\section{METHODS}

\section{Subjects and equipment}

Seven right-handed subjects (ages 22-45), with no history of visuomotor disorders, participated in the experiment. Only one subject $(J C)$ was aware of the experimental design and purpose. All subjects gave informed consent, and the experiment was preapproved by York University's Human Participants Review Subcommittee.

Each subject sat in complete darkness at the center of three mutually perpendicular magnetic fields generated by Helmholtz coils $2 \mathrm{~m}$ in diameter. The torso was constrained by seat belts. We measured the three-dimensional orientation of the right upper arm, the two-dimensional (2-D) gaze direction of the right eye, and the 2-D head direction using search coils (Henriques et al. 1998; Tweed et al. 1990). To ensure that the subjects' pointing was based on vision from the recorded eye, we patched the left eye. Patching makes subjects align the finger with a line joining the target and the viewing eye, even if it is not the dominant eye (Khan and Crawford 2001). Calibration and accuracy were as described previously (Henriques and Crawford 2000; Klier and Crawford 1998).

To obtain visual feedback about head position in some tasks, the subject wore a lightweight bicycle helmet with a laser pointer mounted on top. For the three paradigms where this laser was used, it was continuously on, projecting a faint dot. Before testing, the subject fixated the central light-emitting diode (LED; in front of the right eye) with the helmet on; the laser was adjusted to point at the center target. Using this head-mounted laser as a guide, the subject could point their head in specific directions when required.
Visual targets were red and green LEDs $\left(0.17^{\circ}\right.$ diam and $2.0 \mathrm{~cd} / \mathrm{m}^{2}$ luminance) mounted on a matte black screen located $2 \mathrm{~m}$ from the center of the subject's eye (Fig. 2A). The pointing target, a green LED, was placed at eye level directly in front of the subject's right eye. The orienting targets, red LEDs, indicated the direction for the subject to orient their eye and head or just their head, depending on the task. Nine of these orienting LEDs were placed at $10^{\circ}$ intervals from $40^{\circ}$ left to $40^{\circ}$ right, with the central one vertically adjacent to the green pointing target.

The pointing target was placed at the center to reduce any possible motor effects associated with increasing displacements of the arm (Bock and Eckmiller 1986). However, to ensure that the subject was actively attending to the visual pointing target and not merely making stereotypical, proprioceptively guided movements toward the central direction, we incorporated two nonstandard targets $5^{\circ}$ left and right of center. These were randomly presented in $20 \%$ of the trials. We confirmed that subjects correctly adjusted their pointing for these nonstandard targets but excluded the data for these trials from the analysis.

\section{Experimental paradigms}

BASIC EXPERIMENTAL TASK. There were four paradigms. In all four, the subject pointed as accurately as possible to the location of a pointing target with their right arm and index finger fully extended, and with the head directed toward one of the nine orienting LEDs. Each trial began with the orienting LED illuminated for $1.3 \mathrm{~s}$ to allow the subject time to reposition their head, or both their eyes and head, as the task required. When the orienting target went off, the pointing target was illuminated, and the subject had $2 \mathrm{~s}$ to point to it. Subjects were merely instructed to point accurately at the target; nothing was said about placing the finger on the eye-target line. Each trial ended with an auditory cue (arrow in Fig. 2) signaling to the subject to lower the arm back to the resting position on the arm of the chair next to their hip. In three of the paradigms (all except "Gaze-Deviated without laser"), subjects wore the head-mounted laser; the laser remained on during the pointing response, projecting a faint dot, to help subjects roughly maintain the deviated head position throughout the trial.

CONTROL PARADIGM (FIG. $2 B$ ). To verify that the subject did align the fingertip between eye and target for each position of the head (as assumed in Fig. 1) when visual feedback of the target and hand were available, we had the subject point toward a continuously visible target in dimly lit surroundings. The subject directed the headmounted laser at one of the nine orienting targets. Then the orienting LED was extinguished and the pointing target was illuminated. Maintaining their head position, the subject directed their eye and finger toward the target. The target was on for $2 \mathrm{~s}$ to give the subject time (as established in preliminary tests) and adequate on-line information to move both the eye and arm to it.

GAZE-ON-TARGET PARADIGM (FIG. 2C). This was an open-loop version of the Control paradigm, designed to determine whether similar eye-finger-target alignment occurs in the absence of visual feedback. The pointing target was presented for only $1 \mathrm{~s}$, during which time the subject again looked toward it while maintaining the head posture indicated by the previously flashed orienting LED. Displaying the target for 1,000 ms gave the subject sufficient time to make the eye movement toward it, leaving approximately $500 \mathrm{~ms}$ remaining by the time the final eye position was attained. After the pointing target was extinguished, the subject maintained gaze on its remembered location and pointed there in complete darkness.

GAZE-DEVIATED PARADIGM. In the two Gaze-Deviated paradigms (Fig. 2D) we investigated whether subjects still place the fingertip on the eye-target line without visual feedback and with the gaze line directed away from the pointing target. The subject kept both head and eye directed toward the orienting LED (even after it was extinguished) throughout the trial. The pointing target was flashed for only $500 \mathrm{~ms}$ 

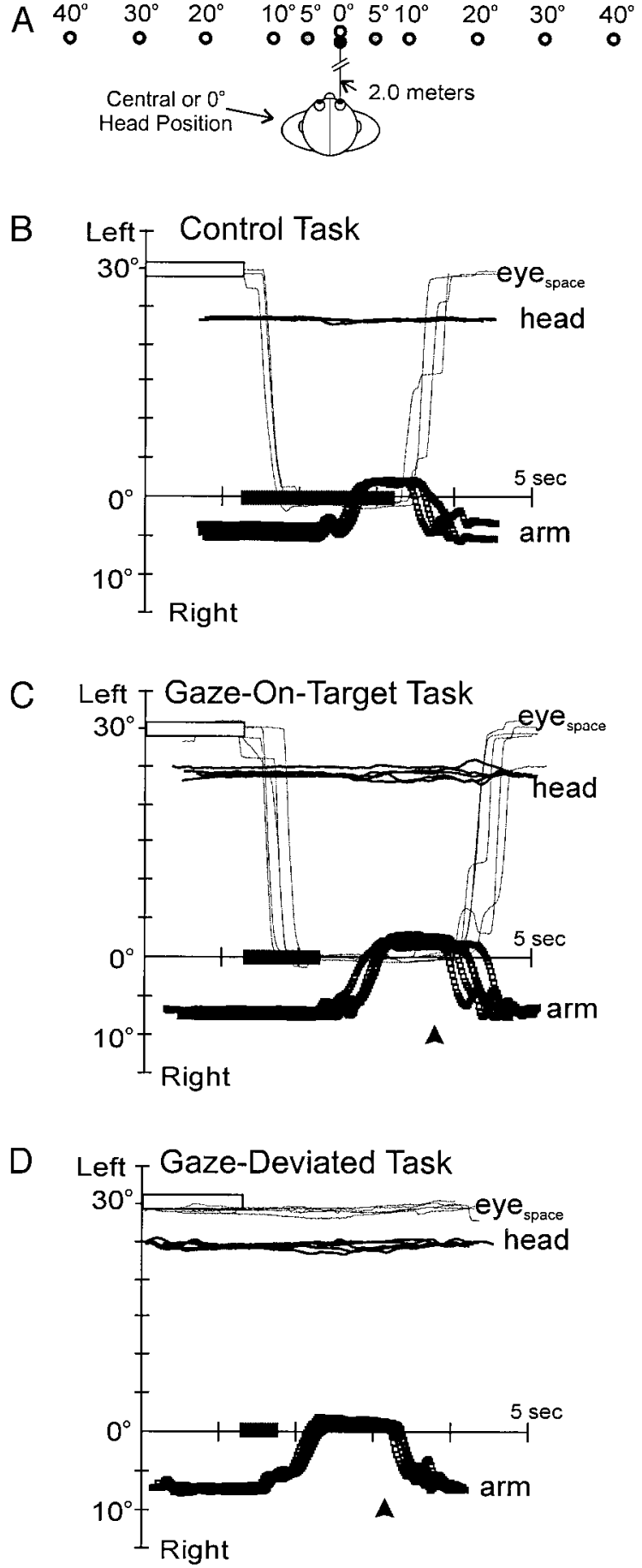

FIG. 2. The 3 main paradigms. A: horizontal placement of red light-emitting diodes (LEDs; open circles) indicating the head-orienting direction and the green LEDs representing the single, main pointing target (black circle) and the nonstandard pointing targets $5^{\circ}$ to the left and right (gray circles), with respect to the subject. $B-D$ : trajectories of the eye, head, and arm for the Control paradigm $(B)$, Gaze-On-Target paradigm $(C)$, and Gaze-Deviated paradigm, when the laser was used $(D)$. Open bars $(\square)$ indicate the location and duration of the illumination of the orienting LED, while the closed bars $(\square)$ represent the same for the pointing target LED. Horizontal position of the eye (thin traces), head (thick traces), and arm (squares) are plotted against time for one subject when the head was oriented toward the $30^{\circ}$ leftward orienting LED. These repositioned movements of the head during the $1.3 \mathrm{~s}$ the orienting LED was on have been omitted for clarity. The traces are taken from 3 trials in the Control task, and 5 trials each from the Gaze-On-Target task and the Gaze-Deviated task. The upward arrows specify the time of the auditory warning signal. because the eyes were not supposed to move toward it. Then the subject pointed toward its remembered location.

GAZE-DEVIATED PARADIGM WITHOUT LASER. This paradigm was the same as the Gaze-Deviated task except that the head-mounted laser was always switched off. With no laser to help aim the head, subjects adopted natural eye-head postures when they fixated the orienting targets.

Each of the three open-loop paradigms was performed 90 times (10 times for each of the 9 orienting LEDs). The orienting LEDs were presented serially from left to right to reduce the time required to adjust head position after each trial. Each set of 10 trials was followed by a 1-s pause to allow subjects time to prepare for the next headorienting LED. The Control paradigm was repeated three times for each head position, for a total of 27 trials.

The paradigms were performed in the following order: 1) GazeDeviated paradigms, 2) Gaze-On-Target paradigm, and 3) Control; so there was no visual feedback until the end. This order was chosen to prevent any learning that might arise if subjects performed those paradigms containing visual feedback or gaze-target alignment prior to those where this possibly confounding information was absent.

\section{Preexperimental paradigms}

To avoid confusion during the experiment, each subject practiced the open-loop paradigms for about 15 min within a day of performing the experiment, but they received no visual feedback regarding their performance. Before the experiment, we localized the centers of rotation of the right eye, head, and arm/shoulder using standard anatomical landmarks (see APPENDIX). The distances and angles between these centers in a horizontal plane (Fig. A1B) were measured (using rulers and protractors) and averaged across all the subjects. We entered these averaged values into an algorithm (see APPENDIX) that calculated the horizontal orientation of the arm that would place the fingertip on the eye-target line for the mean head positions for each of the nine orienting directions recorded during the Control paradigm. From these nine calculated arm angles we subtracted the one calculated for the straight-ahead orienting target, so the arm angles were normalized to $0^{\circ}$ when head position was $0^{\circ}$ (Fig. $1 A$ ). These predicted arm angles were compared with the subject's performance.

\section{Analysis}

Pointing errors in the open-loop paradigms were calculated by subtracting final arm direction from the mean pointing direction for the same head direction in the Control task. In the Gaze-Deviated paradigm without the laser, the head did not face directly toward the red orienting LEDs. We used linear interpolation to compute the arm pointing directions for those head positions in the Control paradigm. Although the range of head positions was smaller in this paradigm, it was large enough in each subject to allow us to test the effect of head position on pointing angle. In tasks where the laser was used to help guide the head, head positions were larger but still less eccentric than that required by the orienting LED. While subjects could aim their head with the laser correctly onto the target when doing so was the only task (Ceylan et al. 2000), they tended to fall short in aiming their head when the main task required them to concentrate on the location of pointing target instead. Because this study looks at only horizontal shifts and rotations, the results discussed in the following text refer to horizontal measurements unless otherwise specified, e.g., horizontal head position, horizontal pointing direction, and horizontal pointing errors.

\section{RE S U L TS}

\section{Predictions for eye-finger-target alignment}

Figure 3 shows the calculated pointing directions $(\diamond)$ that would place the fingertip on the eye-target line, plotted as a 


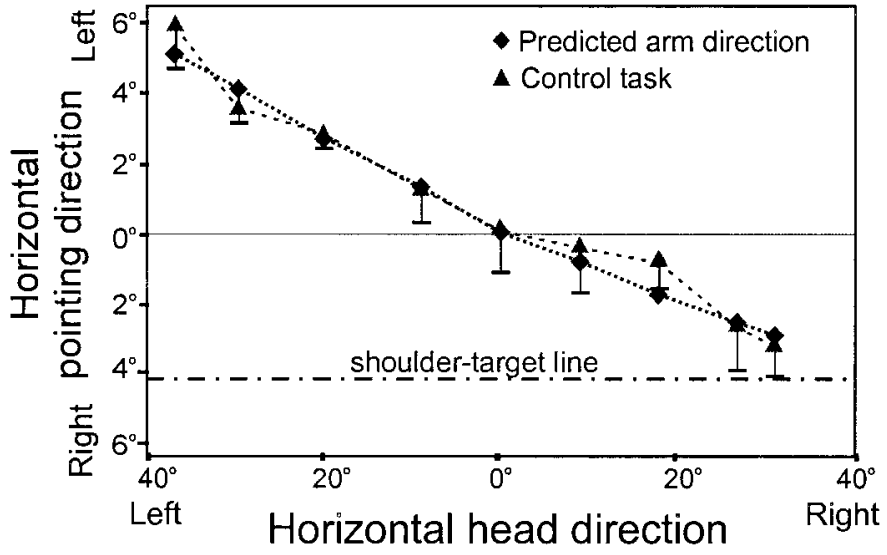

FIG. 3. Horizontal arm position as predicted for ideal eye-finger-target alignment $(\bullet)$ and in the control task ( $\boldsymbol{\Delta}$, averaged across trials and subjects) as a function of mean angular horizontal head position in the control task. The solid line at $0^{\circ}$ represents the arm position for accurate eye-finger-target alignment when head is at center. The flat dotted-dashed line indicates arm direction if subjects oriented their arm for shoulder-target pointing instead. Although within this range of head positions the predicted curve is approximately linear, the curve is inherently nonlinear because as the head moves the eye closer to the right shoulder, the distance between the rotational centers of the eye and the shoulder decreases at a nonlinear rate. Error bars indicate averaged SD across subjects.

function of mean head position during the control task. This curve predicts that as the head turns rightward, arm direction should also progress to the right. For comparison, if the subject simply aligned their arm with the shoulder-target line, then the pointing direction would be fixed and independent of head position, as shown by the dotted-dashed line in Fig. 3.

\section{Closed-loop pointing-control}

Figure 3 shows that subjects do indeed place the fingertip on the eye-target when pointing at a visible target. Triangles $(\boldsymbol{\Delta})$ mark mean pointing directions in the Control paradigm plotted as a function of head position, averaged across trials and then across subjects. The Control curve was nearly identical to the predicted one: as the head moved from left to right, pointing directions for the same target also shifted to the right. The correlation coefficient relating the predicted and Control angles was 0.97 , and the difference between these two data sets was not significantly different from zero (pairwise $t$-test, $t(8)=$ $1.509, P>0.05)$. Thus closed-loop pointing aligns eye, finger, and target even when head repositioning moves the eye.

\section{Open-loop pointing-gaze on target}

Even when the pointing target and hand are no longer visible, subjects still place the finger on the eye-target line. In Fig. 4, the squares (ם) show performance in the Gaze-OnTarget task, where the target was extinguished before the pointing response, averaged across trials and subjects. For comparison, the triangles $(\boldsymbol{\Delta})$ show the Control results from Fig. 3. Apart from a small overall offset, the curves are very similar, with almost identical slopes.

Figure $5 A$ plots the open-loop, Gaze-On-Target performance versus the closed-loop, Control performance for each of the nine head positions for one subject, together with the line of best fit (solid line). Figure $5 B$ shows the slopes for all seven subjects (solid lines) and their average (dashed line). All slopes and their mean $(0.925 \pm 0.242$, mean $\pm \mathrm{SD})$ were close to one (dotted line). This suggests that the CNS compensates for variations in head position about equally well with and without visual feedback.

\section{Open-loop pointing - gaze deviated from target}

The next question is whether gaze direction affects this eye-finger-target alignment strategy. Figure 6 plots the mean performance across all subjects in the Gaze-Deviated paradigms ( $\bigcirc$, with laser; 0 , without laser) versus mean head position for the nine orienting directions. Even here, with both head and gaze deviated from target, the pattern of pointing direction still consistently varied with head position, showing that the finger still landed near the eye-target line, although the curves were somewhat more variable and irregular than in the Control task.

For a clearer comparison, Fig. 7 plots pointing directions in the Gaze-Deviated tasks, with and without laser, versus those in the Control task for corresponding head positions. The left panels display these results and the lines of best fit for one subject. To avoid any distortion in the regression line due to the smaller head displacements in the task performed without the laser, the Control pointing data for that plot were linearly interpolated to match the head positions in the Gaze-Deviated without laser task. The right panels provide the regression lines (solid lines) for all seven subjects. The means (dashed lines) of these 7 slopes were $0.717 \pm 0.223$ (mean \pm SD) with the laser and $0.456 \pm 0.527$ without. These slopes were smaller than those in the Gaze-On-Target task (Fig. 5), significantly so for Gaze-Deviated paradigm without laser as indicated by a pairwise $t$-test, $t(6)=-3.267, P=0.017$. However, a repeated measures two-way ANOVA performed on mean data for individual subjects showed that pointing results differed significantly across head positions $[F(8,80)=27.041, P<0.001]$ but not across the three open-loop paradigms $(P>0.05)$. This result again confirms that under all conditions we tested, subjects systematically redirected the arm as a function of head position so as to bring the pointing finger close to the eye-target line.

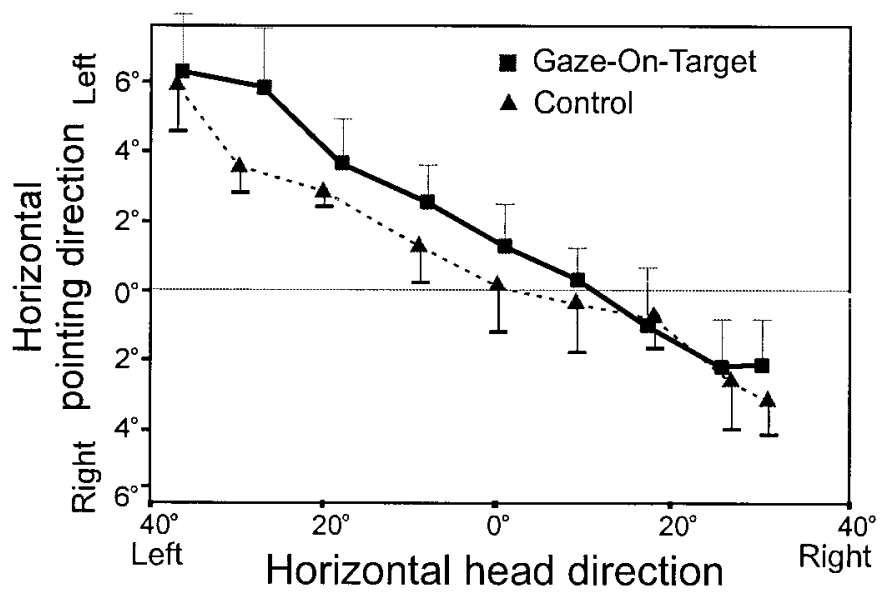

FIG. 4. Final horizontal arm position in the Gaze-On-Target paradigm (ם) as a function of mean angular horizontal head position. For comparison, Control paradigm results $(\boldsymbol{\Delta})$ plotted in Fig. 3 are also included. Error bars indicate averaged SD across subjects. 


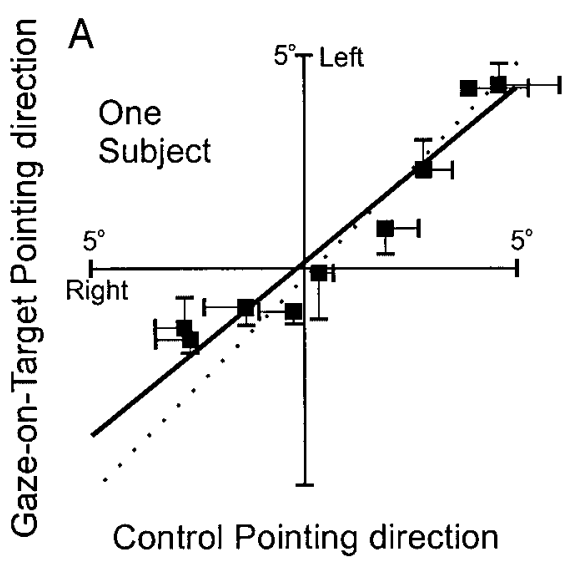

\section{Pointing errors}

Of course the fingertip was not always perfectly aligned with the eye-target line. Analysis revealed a pattern of errors that has been seen earlier in gaze-deviated pointing. We defined pointing errors to be the difference between arm directions in the open-loops tasks and the Control task (see METHODS for further explanation). Figure $8 A$ plots pointing error versus head position for the three open-loop paradigms: Gaze-Deviated paradigms with laser $(\bigcirc)$ and without laser $(\mathbf{)})$, and Gaze-OnTarget paradigm ( $\square$ ). These symbols represent the errors averaged across trials and then across subjects. A zero error means that the fingertip was placed exactly on the eye-target line. The dotted curve shows the errors that subjects would have made if they had simply pointed toward the target as if their head were always centered, with no adjustment for different head postures. Because pointing errors are computed as deviations from Control performance, the dotted line is merely the reverse of the Control curve in Fig. 3.

In the Gaze-On-Target task (ם) subjects tended to point slightly left of the target across all head positions. To clarify the dependence on head position, we removed all such offsets by shifting each curve so that the pointing error for the central head position equaled zero (Fig. 8B). In the Gaze-On-Target task the curve was relatively flat, indicating negligible systematic errors in pointing as the head moved from left to right. In the Gaze-Deviated tasks ( $\bigcirc$, with laser; $\mathbf{O}$, without laser) the

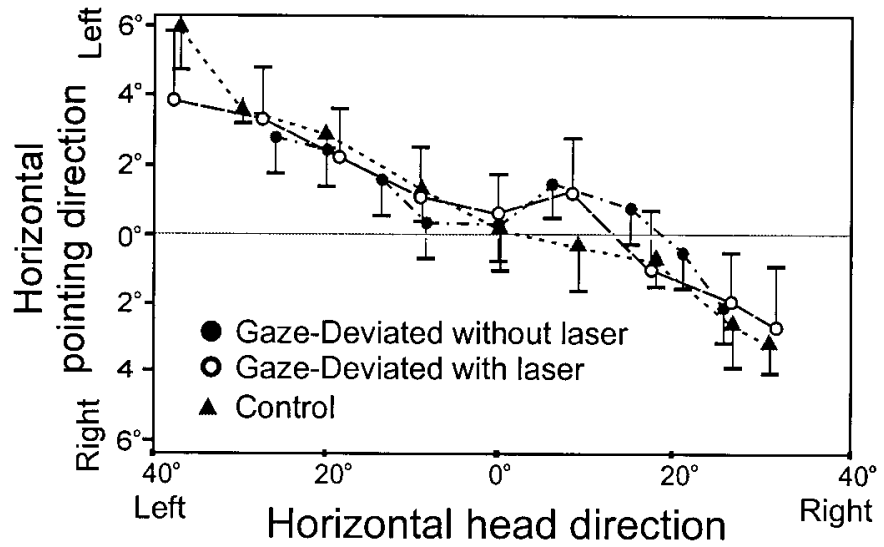

FIG. 6. Final horizontal arm position in the Gaze-Deviated paradigms, with the laser $(\bigcirc)$ and without the laser $(\bullet)$ plotted across the corresponding mean head position (averaged across trials and subjects). For comparison, Control paradigm results $(\mathbf{\Delta})$ plotted in Fig. 3 are also included. Error bars indicate averaged SD across subjects.
FIG. 5. Regression lines fitted to horizontal arm positions in the Gaze-On-Target paradigm (ordinate) as a function of those in the Control paradigm (abscissa). For comparison, a slope of unity (dotted line) is included. $A$ : mean results across trials for each set of head positions ( $\mathbf{\square})$ for 1 subject. $B$ : regression lines for all 7 subjects (solid lines). The dashed line is the average of the 7 individual slopes. mean $r^{2}=0.834$ curves tended to rise to the right, meaning that the pointing finger overshot the eye-target line, landing left of the line when the gaze was to the right, and right when gaze was left. Data from these Gaze-Deviated tasks tended to fall between the curve for Gaze-On-Target pointing errors and the dotted curve, which again represents the pointing errors that would occur if head position were not taken into account. Similar past-pointing errors are seen when subjects point with deviated gaze but the head facing straight ahead (Bock 1986; Enright 1995; Henriques and Crawford 2000; Henriques et al. 1998). To demonstrate this resemblance, we have superimposed, in Fig. $8 B$, pointing errors $(X)$ from one such study (static paradigm: Henriques et al. 1998) for different gaze eccentricities from $30^{\circ}$ left to $30^{\circ}$ right of the central pointing target.

Subjects also made errors in the vertical dimension, pointing below the target by as much as $6^{\circ}$. This vertical undershoot was a consistent finding, occurring for the majority of subjects in open-loop tasks (including tasks in other studies: Henriques and Crawford 2000; Henriques et al. 1998), but it did not vary with the horizontal position of the head or eye.

\section{I S C U S S I O N}

Our results demonstrate the existence within the brain of an accurate internal model of the linkage geometry involved in pointing movements. When pointing at a visual target, our subjects oriented the arm so that the fingertip intersected the line joining target and eye. The geometry of this action is complex - because the rotational centers of the eye, head, and shoulder do not coincide, any head turn shifts the eye-target line-but subjects nevertheless placed the pointing fingertip close to that line, even when they pointed to a target that was no longer visible.

\section{Pointing and linkage geometry}

This performance shows that the brain contains accurate information about arm length and about the rotational centers of the eye, head, and shoulder, including shifts in the eye's center caused by head motion (see APPENDIX for details of the necessary computations). Of course most pointing movements in daily life are performed under visual guidance, but a sophisticated body model of this type would improve motor performance even when visual feedback is available, especially during fast, accurate movements (Desmurget and Grafton 2000; Wolpert and Ghahramani 2000; Wolpert et al. 1995). 


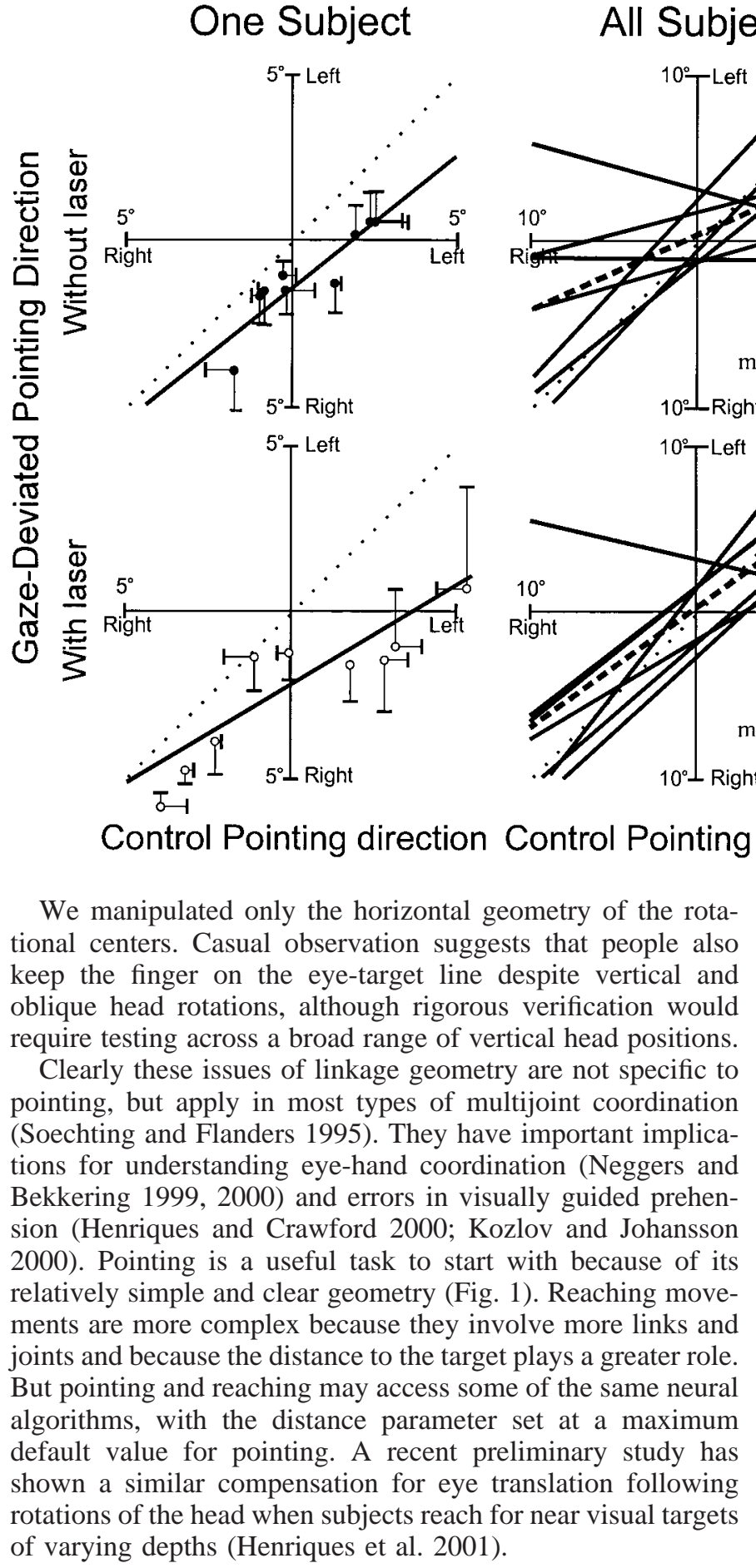

Frames of reference for coding object location with the head free

Many sensory areas in the brain, including superior colliculus and visual cortices, code target locations relative to the eye. A similar eye-fixed frame is used to represent and plan eye and arm movements in parietal cortex (Andersen et al. 1998; Batista et al. 1999; Desouza et al. 2000) and superior colliculus (Stuphorn et al. 2000). But our study shows that information coded in an eye-fixed frame must undergo complex geometric processing to yield appropriate motor commands for arm movements. One way of organizing this processing is described in our computation-on-demand model (Henriques et al. 1998). As proposed in our model, and supported by evidence from a behavioral study by Henriques et al. (1998) and a primate neurophysiological study by Batista et al. (1999), on-line visuomotor representations for arm movements are maintained and updated in an eye-centered frame. However, once a particular target has been selected for immediate action, the retinally coded desired location of the fingertip must then be compared with eye and head position to derive its spatial location with respect to a body-centered frame for muscular control (Caminiti et al. 1998; Flanders et al. 1992; Gnadt et al. 1991; Henriques et al. 1998; Soechting et al. 1990).

Our current study suggests that feedback signals of head orientations are combined with the retinal representation of the target to compute the desired location of the fingertip (as it varies with head posture) in eye-centered coordinates. This computation requires knowledge of the relative location of eye and shoulder, which depend on head position. As shown in the APPENDIX, this computation is simplified in a frame fixed on the eye-target line (which is not necessarily collinear with the gaze line). This could correspond to an intermediate stage in the physiological transformation from eye-centered to shouldercentered representations, i.e., after the retinal target signals have been selected for action. This process may reflect some of the physiological transformations between the parietal and frontal cortices (Batista et al. 1999; Boussaoud and Bremmer 1999; Snyder 2000; Snyder et al. 1998; Van Donkelaar et al. 2000).

\section{Errors generated when gaze is not aligned with target}

When gaze was deviated from the pointing target, subjects tended to past-point, placing the fingertip left of the eye-target 
line when gaze was right of the target, and right of the line when gaze was left. This tendency was not statistically signficant by two-way ANOVA in the present study, but on average it resembles the statistically significant past-pointing seen in other studies of gaze-deviated pointing, both with the headfixed (Bock 1986; Enright 1995; Henriques and Crawford 2000; Henriques et al. 1998) and recently with the head-free (Henriques et al. 2001).

It is not known why subjects past-point when gaze is not on the target. We suggest that people naturally learn to point in such a way that the fingertip will lie on the eye-target line when the head turns toward the target. As shown in Fig. 9, this tendency would lead to past-pointing. When subjects look away from the pointing target (Fig. 9A), the accuracy of pointing may not be clear to peripheral vision. A natural response would be to turn the head and gaze toward the target for a better look. In that case a fingertip positioned on the eye-target line for the deviated head position would not lie on the new eye-target line (Fig. 9B). Subjects might therefore learn to past-point (Fig. 9C) so that the fingertip appeared correctly placed when visually checked. Several studies have demon-
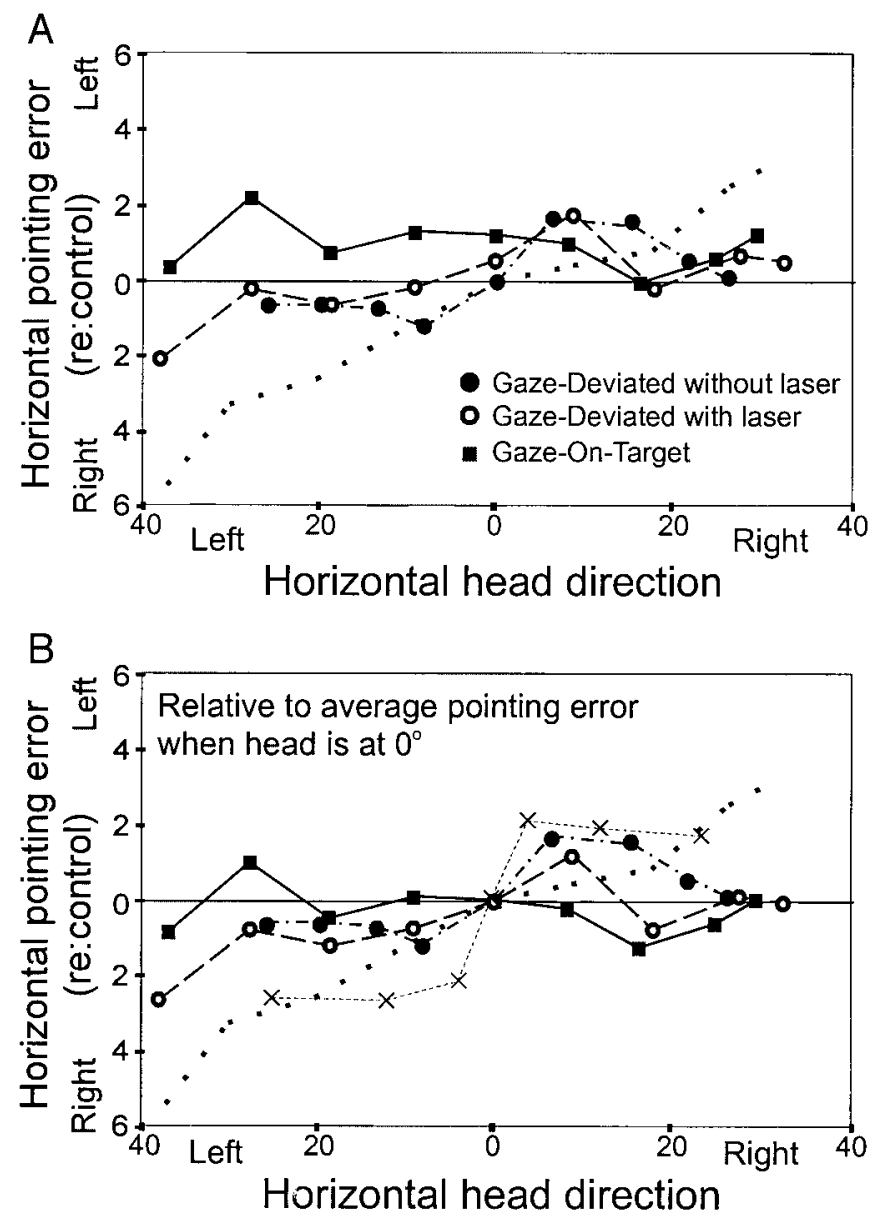

FIG. 8. A: horizontal pointing errors, relative to corresponding control responses, plotted across each set of mean horizontal head positions. GazeOn-Target paradigm ( $\mathbf{\square})$, Gaze-Deviated paradigms: with the laser $(O)$ and without the laser (•). B: same as $A$, except the 3 curves have been shifted so that they are plotted relative to control arm position for the central head posture. Dotted line represents the magnitude of pointing error (re: control) that would occur if subjects maintain the same arm posture (for head center) across all head directions.
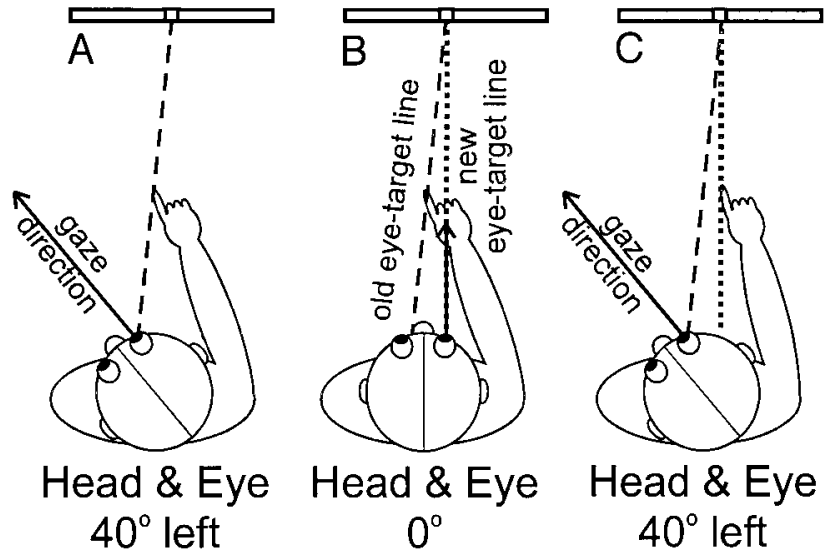

FIG. 9. $A-C$ : the potential errors subjects may make when aligning the fingertip to the eye-target line (dashed line) when both head and eye are deviated $40^{\circ}$ left of center. $A$ : accurate eye-finger-target alignment when gaze is directed $40^{\circ}$ left of target. $B$ : situation where the subject rotated the head and eye back to center $\left(0^{\circ}\right)$ but maintains the previous arm posture for the deviated gaze. Under this condition, the arm would look (to the subject) like it was not correctly positioned because the eye-target line (dotted line) is now shifted with central gaze. $C$ : subjects may learn to compensate for this perceived but spurious error by not shifting arm position as a function of head position when gaze is not aligned with the target, i.e., orienting the arm so that it falls not on the eye-target line produced when both the eye and head are aligned with target direction (dotted line).

strated that false visual feedback can recalibrate motor commands (Flanagan and Rao 1995; Ghahramani et al. 1996; Shadmehr and Moussavi 2000; Wolpert et al. 1994, 1995), altering goal-directed movements even outside the region of spurious feedback. The existence of past-pointing underscores the importance of maintaining gaze on target for accurate eye-hand coordination (Kozlov and Johansson 2000; Neggers and Bekkering 1999, 2000).

\section{A P P E N D I X}

\section{Pointing geometry}

Before each experiment we measured the locations, in the horizontal plane, of the centers of rotation of the subject's right eye, head, and right shoulder. The eye's center of rotation is approximately the center of the orbit, $1.6 \mathrm{~cm}$ behind the cornea (Howard 1982). To find the center of rotation of the head, we had subjects wear a grid-patterned cap; a laser light, projecting downward, was adjusted so that it illuminated the spot on the cap that did not move when the subject swung their head horizontally. Similarly, the shoulder's center of rotation was the site (on its upper surface, near where the line of the long axis of biceps met the midpoint of the acromion) that did not move when the subject swung their arm horizontally.

Figure A1 shows the centers of rotations of the eye, head, and shoulder (-) and variables necessary for computing the arm angle that aligns the fingertip with the eye-target line (dark dashed lines). In this schematic, the head is oriented $40^{\circ}$ to the left during pointing, as shown in Fig. A1A. In $C$ and $D$, three vectors (dotted lines) join the centers of rotation and the finger: $a$ is the vector of the extended arm, from the shoulder to the fingertip; $c$ (for "clavicle") is the vector from the head to the shoulder; and $r$ is the vector from the right eye to the center of rotation of the head. Using a ruler and protractor, we found the $x$ - and $y$-coordinates of these three fixed-length vectors, $r, c$, and $a$, when the head was pointing forward. Their mean lengths were 9.4 $\mathrm{cm}( \pm 1.7, \mathrm{SD})$ for $r, 19.8 \mathrm{~cm}( \pm 1.9)$ for $c$, and $72.6 \mathrm{~cm}( \pm 4.5, \mathrm{SD})$ for $a$. When the head turns, $c$ remains fixed in space because the torso 
A
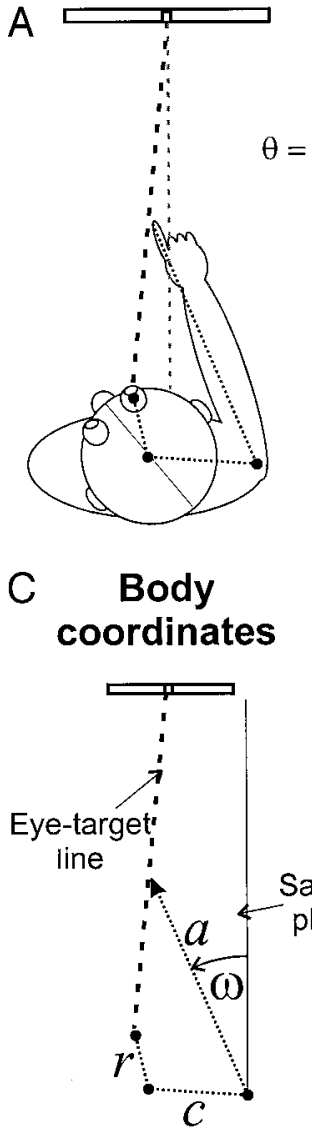

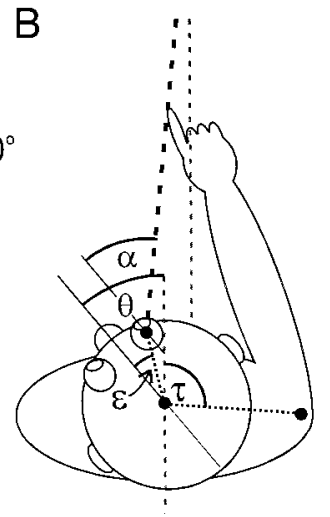

D Eye-target coordinates

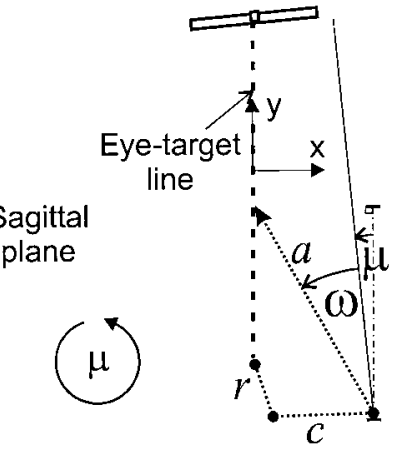

FIG. A1. A schematic of the parameters used to compute ideal horizontal arm position for correct eye-finger-target alignment. $A$ : the head is oriented $40^{\circ}$ left of center. Filled circles mark the rotational centers of the eye, head, and shoulders. $C$ and $D$ : the vectors (dotted lines) connecting EYEcr to HEADcr, HEADcr to SHOULDERcr, and SHOULDERcr to fingertip are labeled $r, c$, and $a$, respectively, as in $A$ with the caricature removed. The intersecting fingertip is indicated by the arrowhead of vector $a$. The eye-target line is indicated by a dashed line, while the thin solid line marks the sagittal plane of the torso. $C$ : the $y$-axis is parallel to the sagittal plane. Ideal arm angle from a sagittal reference plane (thin vertical line) is indicated by $\omega . D$ : the vectors are rotated into a new reference frame where the $y$-axis is no longer the sagittal plane but rather the eye-target line (dashed line). In this frame, the fingertip lies on the $y$-axis and the $x$-components of $r^{\prime}, c^{\prime}$, and $a^{\prime}$ add up to zero, so that $a_{x}^{\prime}=-r_{x}^{\prime}-c_{x}^{\prime}$. The magnitude of rotation between the 2 reference frames is indicated by $\mu$ : the angle between the sagittal plane and the eye-target line. $B$ : additional variables measured either prior to or during the experiment: $\epsilon$, the angle between $r$ and head mid-line; $\tau$, the angle of the $c$ from the torso mid-line; $\alpha$, eye-in-head position; and $\theta$, head rotation from center.

has not moved (mean angle of $103 \pm 4.0^{\circ}$ from midsagittal line), but $r$ rotates with the head. Therefore the brain must rotate $a$ to realign the fingertip with the eye-target line. To assess its accuracy, we computed the arm angle $\omega$ that would yield perfect eye-finger-target alignment.

To do this, we rotated the vectors $r$ and $c$ by $\mu$ into a new coordinate system where the $y$-axis is no longer the sagittal plane of the torso but rather the line joining the eye and the target (dark dashed lines in Fig. A1). The computation could have been done in a body-fixed frame, but it is simpler in these eye-target coordinates. The angle $\mu$ for the coordinate rotation is the angle between the eye-target line and the sagittal plane of the torso (the dark dashed line and light dashed line, respectively, in Fig. A1A). The constants and variables used to compute $\mu$ include $d$ which is the distance from the EYEcr to the target when the head points forward (length of light dashed line in Fig. A1A), $|r|$ which is the length of $r$, and $\epsilon$ as the angle between $r$ and the sagittal plane of the head (mean of $26.5 \pm 2.9^{\circ}, \mathrm{SD}$ ). We let $\theta$ be the head's angle of rotation away from straight forward, measured by

the search coils. Then $\mu=\tan ^{-1}\{|r|[\sin \epsilon+\sin (\theta-\epsilon)]\} /\{d+$ $|r|[\cos \epsilon-\cos (\theta-\epsilon)]\}$. Incidentally, these values are likely to be available to the CNS, for example: depth perception $(d)$, retinal stimulus location + an internal sense of eye position $(\alpha)$ and an internal sense of head position $(\theta)$.

We then computed the arm vector that would place the fingertip on the eye-target line, which is the $y$-axis in eye-target coordinates. Clearly the fingertip lies on the $y$-axis if the $x$-components of $r, c$, and $a$ (all in eye-target coordinates) add up to zero; that is, $a_{x}$ must equal $-r_{x}-c_{x}$. Using some trigonometry, not shown here, these $x$-components of $r, c$, and $a$ can be easily derived from the angles illustrated in Fig. A $1 B$ (see legend). The $y$-component of $a$ is then determined, because the length of the arm is constant and equals, by Pythagoras's theorem, the square root of $a_{x}^{2}+a_{y}^{2}$; that is, $a_{y}$ is the positive square root of (arm length squared $-a_{x}^{2}$ ). The angle between the vector $a$ and the eye-target line is then $\tan ^{-1}\left(-a_{x} / a_{y}\right)$. Finally, the desired angle between the arm and the coronal plane of the torso is $\omega=$ $\tan ^{-1}\left(-a_{x} / a_{y}\right)-\mu+\pi / 2$.

We thank A. Constantin, E. Klier, J. Martinez, P. Medendorp, M. Niemeier, and $\mathrm{D}$. Tweed for constructive criticism on the manuscript.

This work was supported by grants from the Canadian Natural Sciences and Engineering Research Council. D.Y.P. Henriques is supported by an E. A. Baker Foundation-Canadian Institutes of Health Research Doctoral Research award. J. D. Crawford is supported by the Canadian Research Chair Program.

\section{REFERENCES}

Andersen RA, Snyder lH, Batista AP, Buneo CA, and Cohen Ye. Posterior parietal areas specialized for eye movements (LIP) and reach (PRR) using a common coordinate frame. Novartis Found Symp 218: 109-122, 1998.

Batista AP, Buneo CA, Snyder LH, and Andersen RA. Reach plans in eye-centred coordinates. Science 285: 257-260, 1999.

BосK O. Contribution of retinal versus extraretinal signals towards visual localization in goal-directed movements. Exp Brain Res 64: 476-482, 1986.

Воск O. Localization of objects in the peripheral visual field. Behav Brain Res 56: 77-84, 1993.

BOCK O AND ECKMILLER R. Goal-directed arm movements in absence of visual guidance: evidence for amplitude rather than position control. Exp Brain Res 63: 451-458, 1986.

BoussaOud D AND BREMMER F. Gaze effects in the cerebral cortex: reference frames for space coding and action. Exp Brain Res 128: 170-180, 1999.

CAMINITI R, FERRAINA S, AND MAYER AB. Visuomotor transformations: early cortical mechanisms of reaching. Curr Opin Neurobiol 8: 753-761, 1998.

Ceylan M, Henriques DYP, Tweed DB, and Crawford JD. Task-dependent constraints in motor control: pinhole goggles make the head move like an eye. J Neurosci 20: 2719-2730, 2000.

DESMURGET M AND GRAFTON S. Forward modeling allows feedback control for fast reaching movements. Trends Cogn Sci 4: 423-432, 2000.

Desouza JF, Dukelow SP, Gati JS, Menon RV, Andersen RA, and Vilis T. Eye position signal modulates a human parietal pointing region during memory-guided movements. J Neurosci 20: 5835-5840, 2000.

ENRIGHT JT. The non-visual impact of eye orientation on eye-hand coordination. Vision Res 35: 1611-1618, 1995.

FLANAGAN JR AND RAO AK. Trajectory adaptation to a nonlinear visuomotor transformation: evidence of motion planning in visually perceived space. $J$ Neurophysiol 74: 2174-2178, 1995.

Flanders M, Helms Tillery SI, and Soechting JF. Early stages in a sensorimotor transformation. Behav Brain Sci 15: 309-362, 1992.

GHAHRAMANi Z, WolPERT DM, AND JoRDAN MI. Generalization to local remappings of the visuomotor coordinate transformation. J Neurosci 16: 7085-7096, 1996.

GNADT JW, BRACEWELl MR, AND ANDERSEN RA. Sensorimotor transformation during eye movements to remembered visual targets. Vision Res 31: 693$715,1991$.

Helms Tillery SI, Flanders M, and Soechting JF. A coordinate system for the synthesis of visual and kinesthetic information. J Neurosci 11: 770-778, 1991.

HENRIQUes DYP AND CRAWFORD JD. Direction-dependent distortions of retinocentric space in the visuomotor transformation for pointing. Exp Brain Res 132: 179-194, 2000. 
Henriques DYP, Klier EM, Smith MA, Lowy D, and Crawford JD. Gaze-centered remapping of remembered visual space in an open-loop pointing task. J Neurosci 18: 1583-1594, 1998.

HenRiques DYP, MEdendorp WP, Gielen CCAM, and CRAWFord JD. The eye-hand coordination system accounts for head orientation and target depth during reaching toward near targets. Soc Neurosci Abstr 27: 940.10, 2001.

Howard IP. Human Visual Orientation. New York: Wiley, 1982.

Khan AZ AND CRAwFord JD. Coordinating one hand with two eyes: gazedependent reversal of ocular dominance in a pointing task. Soc Neurosci Abstr 27: 940.12, 2001.

KLIER EM AND CRAWFORD JD. The human oculomotor system accounts for 3-D eye orientation in the visual-motor transformation for saccades. $\mathrm{J} \mathrm{Neu}$ rophysiol 80: 2274-2294, 1998.

KoZLOV Y-T AND JOHANSSON R. Role of eye movements in control of bimanual manipulatory task. Soc Neurosci Abstr 26: 179, 2000.

NEGGERS SFW AND BEKKERING H. Integration of visual and somatosensory target information in goal-directed eye and arm movements. Exp Brain Res 125: 97-107, 1999.

NegGers SFW and BekKering H. Ocular gaze is anchored to the target of an ongoing pointing movement. J Neurophysiol 83: 639-651, 2000.

Prablanc C, Pelisson D, and Goodale MA. Visual control of reaching movements without vision of the limb. I. Role of retinal feedback of target position in guiding the hand. Exp Brain Res 62: 293-302, 1986.

Rossetti Y, Stelmach G, Desmurget M, Prablanc C, and Jeannerod M. The effect of viewing the static hand prior to movement onset on pointing kinematics and variability. Exp Brain Res 101: 323-330, 1994.

SHADMEHR R AND MousSAVI ZM. Spatial generalization from learning dynamics of reaching movements. J Neurosci 20: 7807-7815, 2000.
SNYDER LH. Coordinate transformations for eye and arm movements in the brain. Curr Opin Neurobiol 10: 747-754, 2000.

SNyder LH, Grieve KL, Brotchie P, And ANDERSEn RA. Separate body- and world-referenced representations of visual space in parietal cortex. Nature 394: 887-891, 1998.

SOEChTING JF AND Flanders M. Psychophysical approaches to motor control. Curr Opin Neurobiol 5: 742-748, 1995.

Soechting JF, TILlery SI, AND Flanders M. Transformation from head-toshoulder centered representation of target direction in arm movements. J Cogn Neurosci 2: 32-43, 1990.

Stuphorn V, Bauswein E, AND Hoffmann KP. Neurons in the primate superior colliculus coding for arm movements in gaze-related coordination. J Neurophysiol 83: 1283-1299, 2000.

Tweed D, Cadera W, and Vilis T. Computing three dimensional eye positions quaternions and eye velocity from search coil signals. Vision Res 30: 97-110, 1990.

Van Donkelaar PV, Lee JI-H, And Drew AS. Transcranial magnetic stimulation disrupts eye-hand interactions in the posterior parietal cortex. $\mathrm{J} \mathrm{Neu}$ rophysiol 84: 1677-1680, 2000.

Van Donkelaar PV and Straub J. Eye-hand coordination to visual versus remembered targets. Exp Brain Res 133: 414-518, 2000.

WOLPERT DM AND GHAHRAMANi Z. Computational principles of movement neuroscience. Nature Neurosci 3: 1212-1217, 2000.

Wolpert DM, Ghahramani Z, and Jordan MI. Perceptual distortion contributes to the curvature of human reaching movements. Exp Brain Res 98: 153-156, 1994

WOLPERT DM, GHAHRAMANi Z, AND JoRDAN MI. An internal model for sensorimotor integration. Science 269: 1880-1882, 1995. 